\title{
The clinical features of herpes simplex encephalitis (acute necrotizing encephalitis)
}

\author{
L. A. LiverSEDGE \\ Department of Clinical Neurology, Manchester Royal Infirmary, Manchester M13 9WL
}

THE descriptive literature of the encephalitides of all types is characterized by a recognition both of the pleomorphic nature of the disease and of our total therapeutic helplessness. It follows, therefore, that in the past a diagnosis of encephalitis tended to be something of an academic exercise, fatalistically imprecise and the sort of disorder liable to give neurology a bad name. There is now a growing feeling that it may be possible to influence favourably the course of the encephalitides and this, of course, makes precise diagnosis absolutely imperative, particularly if the potentially therapeutic agents are also potentially harmful to the patient.

An important feature of the direct viral encephalitides is that the virus and the brain cell fuse their metabolic needs (the process was formerly described in euphemistic and euphonious terms as obligate cellular parasitism) and so the anti-viral agent is liable to prove harmful to the cells which it is initially designed to help. For these reasons an absolute clinical guide to the diagnosis of the herpes encephalitis (or for that matter any other type of encephalitis) would be ideal. Unfortunately such clinical diagnostic absolutism does not exist, but one hopes as far as possible to delineate as clearly as may be possible such features in encephalitis as may lead to the identification of the infective agent and so to its potential destruction.

It must be emphasized at the outset that a conclusive aetiological diagnosis can be made in life only with the aid of brain biopsy. Opinions on the advisability of brain biopsies vary and furthermore opinions vary on the site in which brain biopsies should be made. It is extremely interesting to read the varying views on the diagnostic possibilities, beginning with one American school, whose opinions may be summed up in a quotation of an editorial of the Journal of the American Medical Association, 4 March 1968.

'The clinical features of herpes encephalitis in the adult are often distinct from those induced by other infecting agents.... Patients manifest personality changes, bizarre behaviour, hallucinations and aphasia in the early stages of the disease, suggesting a unique localization of the infection in the temporal lobe. This clinical constellation is sufficiently unusual to suggest that the aetiological agent is the herpes virus .... if treatment is to be considered a definitive diagnosis should be sought from biopsy. There are few justifications for this procedure, but this may prove to be one.'
Two points of clinical interest should be made about this statement. Firstly, it makes no reference to any epileptic symptomatology-a common clinical feature of herpes encephalitis, and secondly, defines a syndrome, which must give rise to radiological and surgical assays to establish the presence or absence of a localized abscess, as all the features alluded to above may occur in such a condition. Adams \& Jennett (1967) described their experience with eight cases which proved to be encephalitic in origin, in which the features suggested intracranial expanding lesions of the type found in abscesses. Six of these cases died, despite all efforts to help them and the remaining survivors were in a very unsatisfactory clinical state.

A rather more realistic, if more modest approach, was made by Bergouignan et al. (1968), who declared that 'clinical diagnosis on the other hand is exceedingly difficult' and correctly indicated the extraordinarily variable features, which may be produced when the herpes virus affects the brain. These recognized clinical difficulties may be illustrated by three of our own recent cases.

\section{Case 1. D.M., male, aged 35}

On 23 April 1971 this patient developed a flu-like illness with some headache and was treated with tetracycline. Seven days later he became unduly sleepy and was admitted to Burnley Hospital, where he was found to be drowsy, had meningitic signs and showed slight pyramidal signs. On 1 May 1971 he had a series of convulsions, followed by confusion and dysphasia. Cerebro-spinal fluid analysis at that time showed 9 cells with a protein of $40 \mathrm{mg}$. On 3 May 1971 because of his continuing deterioration he was transferred to the Manchester Royal Infirmary, where on arrival he was found to be facile, disinhibited and variably dysphasic. An electroencephalogram carried out at that time showed diffuse high voltage delta activity, but there was no asymmetry or focal abnormality. On the day of his admission herpes simplex virus was isolated from his throat. On 6 May 1971 he developed status epilepticus with right-sided focal manifestations, then coma, when the right-sided pyramidal signs became pronounced. A left carotid angiogram was performed and proved to be perfectly normal. Shortly after this he had a left frontal biopsy and immediately treatment was begun with cytarabine, $300 \mathrm{mg}$ intravenously daily-a dose which was continued for the next 6 days. On 7 May 
1971 herpes was isolated from the brain biopsy. On that day too he again went into right-sided focal status epilepticus, which responded to treatment with valium and epanutin. On the following day his condition was remarkably improved and on 11 May 1971,5 days after beginning treatment, he was conscious, but exceedingly garrulous and disinhibited and given to a good deal of obscene language. The improvement, however, continued and his social behaviour fortunately improved. On 17 May 1971 his condition was complicated by the fact that his platelet count fell to $25,000 / \mathrm{mm}^{3}$ and his haemoglobin fell to $10 \mathrm{~g} / 100 \mathrm{ml}$. There was also agranulocytosis. His bone marrow showed depression of all elements and so on the advice of Dr Delamore he was given oxymethaline, $50 \mathrm{mg}$ t.d.s. During the next few days both his encephalitic condition and his blood condition improved, so that by 26 May 1971 his blood count was normal and on 18 June 1971 he was able to return home.

A curious feature of the post-encephalitic state was that he had a memory gap of about 2 years, but this has gradually filled in. He also had some intellectual impairment, as tested by formal psychometric methods. In the intervening period he has steadily improved. He has returned to work but it has to be recorded that he has had six nocturnal epileptic attacks in that time, the last one taking place some 2 months ago. When last seen his physical condition was excellent, though he still had a rather patchy memory gap, extending over the last 2 years. His wife, who is a shrewd observer, says that as far as she was concerned he had returned to normal, apart from this memory gap.

In summary, therefore, here was a patient who presented with clouding of consciousness, bizarre behaviour, variable dysphasia, very definite epileptic signs and symptoms and focal right-sided signs, but with a normal angiogram and a diffuse non-localized electroencephalographic abnormality. Another clinical feature of note was the meningitic picture, even though the cerebro-spinal fluid was not very abnormal.

\section{Case 2. J.F., female, aged 51}

This lady really had a remarkable clinical history, for in 1962 she was admitted to a hospital in the North, complaining of abdominal pain. Following investigation, laparotomy revealed that she had polycystic kidneys. In October 1969 she was transferred to Withington Hospital for the fitting of a Brescia shunt, since she had developed a pronounced uraemic state. This, apparently, was quite successful and she was in reasonable health until 1971. In June 1971 she was admitted and a successful kidney transplant was carried out. In September 1971, however, she developed a small urinary fistula from the transplant wound and so had to be readmitted. Clinical re-examination showed that she was rather depressed, in addition to her biochemical disorder, but it was considered that her renal function was well-maintained. The fistula was quite small and there was very little leak. An intravenous pyelogram showed a collection of urine around the ureteric anastomosis. Her depression appeared to respond rather poorly to amitriptyline, but she made quite good progress and became ambulant. On 27 October 1971, however, she became very confused, had variable dysphasia, developed tremors with myoclonus and gradually declined.

Clinical examination on 10 November 1971 showed that the abnormalities comprised dyspraxia, dysphasia and generalized tremors, rather more pronounced on the right than the left, with cogwheel rigidity in the right arm and leg. She had curious myoclonic jerks and a tendency to carphology. All her reflexes were depressed; her right plantar response was extensor, the left was flexor. It was felt the clinical picture here was that of a diffuse encephalopathy and it was suggested that there might well be a viral infective agent. The cerebrospinal fluid showed no significant abnormality, but her electroencephalogram showed diffuse slow-wave activity.

A brain-scan showed an area of doubtful activity in the right frontal region. Unfortunately, she died on 13 November 1971. By this time bone marrow culture had yielded a growth of a herpes simplex virus. Unfortunately no brain tissue could be obtained since permission for necropsy was not granted. It seemed, however, with herpes virus in the bone marrow, that it was very likely that the whole process was due to herpetic encephalitis.

Again the encephalitic picture was that of a generalized confusional state, with dysphasia, myoclonic type epileptic manifestations, right-sided pyramidal signs, some extra-pyramidal manifestations and of course the obvious behavioural disorder. In this case there was no evidence of meningeal affection.

\section{Case 3. O.B., female, aged 21}

In June 1971, this girl became pregnant by a married man. The pregnancy was accepted, the family background was good, the daughter's mental health was apparently very stable and it was intended that she should marry the father of the child as soon as he got his divorce. On 6 October 1971 she began to be a little drowsy, she had a little difficulty in speaking and the following day saw her general practitioner, who arranged for her to be seen as part of a routine follow-up at the Maternity Hospital. She then went to her work where apparently she was "not speaking' and so was sent home. Later that day she was seen at the Maternity Hospital and referred for a 
psychiatric opinion because of her strange behaviour and the difficulty with her speech. The following day she was seen at Prestwich Hospital and since it was considered that this was some form of reactive depression associated with her pregnancy, she was actually given one electro-convulsive treatment. Following this, although she recovered from the anaesthetic she sank into coma and so was transferred to the Manchester Royal Infirmary. On arrival here she was comatose and flaccid, having had focal epileptic movements in the right arm and leg with occasional myoclonic jerks on the left. From time to time both legs appeared to jerk to some extent. 2 days later it was noted that she was exhibiting single convulsive movements occurring synchronously in the left face, hand and left arm and occasionally in the right arm. Her reflexes were all rather brisk and the plantar responses were mute.

Cerebro-spinal analysis showed 144 lymphocytes with a protein of $30 \mathrm{mg}$. Isotope brain-scan showed a suspicion of a localized area of abnormal activity in the left posterior temporal region. Electroencephalogram in this case was interesting. The background rhythm was that of very, very slow delta activity, ranging from 0.5 to 3 cycles/sec, but associated with this generalized slow activity there were sharp periodic waves of the type described by Upton \& Gumpert (1970). On 13 October 1971 a brain biopsy was carried out which later proved to be positive for herpes simplex virus, but unfortunately the patient died untreated on the morning of 15 October 1971.

In summary, a relatively short history of stupor, abnormal behaviour, dysphasia going on to aphasia and finally epileptic manifestations.

It will be seen from these cases that the assertion in the Journal of the American Medical Association (1968) does not really cover the whole subject of accurate clinical diagnosis, for the picture can be extremely variable. Miller, Hesser \& Tompkins (1966) reviewed twenty cases of encephalitis, in ten of whom a definite diagnosis of herpes simplex encephalitis had been made, six at necropsy, and four on the basis of $A$ type intranuclear inclusion bodies in biopsy specimens; in the remaining ten cases the diagnosis was considered to be established by the clinical picture and a fourfold rise in herpetic antibodies during the course of the illness. Miller et al. (1966) make a very important point that despite the most careful search in no case could the virus be isolated from the cerebrospinal fluid and it seems that very few authorities have been successful in this search. Miller also stresses the very poor prognostic implications of coma and convulsions, though it is to be noted that both these features were seen in our patient, T.M., just before treatment with cytarabine was begun.

Bergouignan et al. (1968), recognized these clinical difficulties in diagnosis, but then went on to attempt a categorization of various forms of herpetic encephalitis on the rather slender basis of five cases of their own added to a survey and analysis of other cases in the literature. Although it might be said that this is too obviously an attempt to tidy up reality the Bergouignan clinical analysis has much to commend it from the practical point of view, since it does emphasize the extraordinary clinical variations which may be seen in this condition.

\section{Classification}

Type I: Meningo-encephalitic. This is characterized by stupor, convulsions, meningeal reaction with a cerebro-spinal fluid pleocytosis of up to $200 \mathrm{lym}$ phocytes. The prognosis is poor.

Type II: The Prolonged Type. An acute attack lasting 10-14 days is followed by apparent spontaneous improvement, but then a relapse occurs 6-8 weeks later. Again the prognosis is poor.

Type III: Neurosurgical. This presents as a temporal lobe space-occupying lesion with focal electroencephalographic abnormalities and displacement of the middle cerebral vessels on angiography. Surgical treatment may prove beneficial, but there is usually an appreciable residual deficit and the prognosis overall is poor. This, of course, is the type of disorder described by Adams \& Jennett (1967), and also by Cope \& Howieson (1967). It is to be noted that the Adams cases also showed a considerable lymphocytosis in the cerebro-spinal fluid. Six of the eight cases, as mentioned above, died and the other two were in rather poor condition despite the surgical aid.

Type IV: 'The Curable Form'. In these cases spontaneous recovery takes place, but there is a considerable residual deficit. Bergouignan speculates on the possibility that the relatively early development of viral antibodies in the patient may be responsible for the degree of recovery, but, in fact, no case of his own followed this particular course.

\section{Radiological features}

It will be apparent from the above review of the problem that the lesions in the brain tissues are very widespread. In certain cases, however, it may be that the areas in which the most rapid growth of virus is taking place, or else in which the most intense antiviral antibody activity is occurring, may respond with localized oedema and so give rise to the appearance of a space-occupying lesion. In such cases the angiographic appearances are those of vascular shift, without evidence of tumour circulation and in a number of recorded cases this particular picture has been seen predominantly in the temporal lobe, though it must be added that other areas of the brain have been affected. 
Brain-scan gives somewhat similar evidence of local increase in activity of a non-specific type. One clinical advantage of demonstrating such a picture is that it does give a clue to the site at which a positive brain biopsy is likely to be obtained, but on the other hand, if the radiological lesions appear to be in a dominant temporal lobe many neurologists would not be in favour of exposing the already damaged tissue to the hazards of brain biopsy. On the other hand such a diagnostic procedure will rule out the alternative possibility of a cerebral abscess.

\section{Electroencephalographic findings}

As in the case of the radiological findings, the electroencephalogram varies enormously. Yet there seem to be certain clearly definable features in certain cases; thus Rawls et al. (1966) in discussing five cases of their own refer to 'generalized, lateralized, or localized slow waves with irritation waves in the form of sharp spikes'. Upton \& Gumpert (1970) are rather more definitive in their conclusions and present four main characteristics which they have assembled from the records of six cases of their own and a review of fifteen others. The features which they stress are: (1) a slow-wave background; (2) periodic complexes 'between the second and fifteenth days of the illnesses'; (3) disappearance of these complexes without a parallel improvement in the clinical state of the patient; (4) in certain cases, temporal or fronto-temporal localization of the electroencephalographic abnormalities.

The periodic complexes to which they allude seem to be similar to the sharp irritation waves mentioned in the earlier paper by Rawls. It is to be noted that in our third case these waves were also to be seen in association with the slow-wave background. It should be added, however, that other authorities, e.g. Scott \& Prior (1970) and Adams \& Jennet (1967) are less convinced about the specificity of these periodic changes. One can conclude, however, that the electroencephalographic picture in general is very variable, yet in certain particular cases, clearly delineated abnormalities may be seen and thus give support to the clinical diagnosis and perhaps afford an additional reason for carrying out brain biopsy.

\section{Summary and Conclusions}

The clinical picture of herpes simplex encephalitis is like that of all encephalitides, expectedly very variable. On the other hand, there are some important clinical indicators which if present make the diagnosis extremely probable and thus lead to brain biopsy and the concurrent administration of antiviral agents. These basic indicators may be listed as follows:

(1) Clinical. Variable clouding of consciousness, personality change, bizarre behaviour, speech disorder and epileptic features of one sort or another (an essential negative factor is that there should be no potential source of pyogenic infection to suggest the alternative diagnosis of brain abscess).

(2) Cerebro-spinal fluid. Lymphocytic pleocytosis.

(3) Electroencephalogram. Diffuse slow-wave activity with or without localization, associated periodic 'irritation' waves, particularly from the second to fiftieth day of the encephalitic illness.

(4) Angiography. An appearance of a spaceoccupying lesion, particularly of the temporal lobes, with no tumour vascular pattern. Paradoxically if angiography is normal in the presence of all the other features, then the herpetic diagnosis becomes even more probable.

\section{References}

AdAms, J. H. \& JennetT, W. B. (1967) Acute necrotizing encephalitis: the problem of diagnosis. Journal of $\mathrm{Neu}-\mathrm{I}$ rology, Neurosurgery and Psychiatry, 30, 248.

Bergouignan, M., Julien, J., Vital, C. \& Duvert, M. (1968) Notre expérience de l'encéphalite aigüe nécrosante. Presse Médicale, 709.

Cope, V. \& Howieson, J. (1967) Radiological findings in acute necrotizing encephalitis due to herpes simplex virus. Clinical Radiology, 18, 109.

EDITORIAL (1968) Diagnosis of herpes simplex encephalitis. Journal American Medical Association, 203, 882.

Miller, J. K., Hesser, F. \& Tompkins, V. N. (1966) Herpes simplex encephalitis: report on 20 cases. Annals of Internal Medicine, 64, 92.

Rawls, W. E., Dyck, P. J., Klass, K. W., Greer, H. D. \& HermanN, E. C. (1966) Encephalitis associated with herpes simplex virus. Annals of Internal Medicine, 64, 104.

ScOTT, D. F. \& PRIOR, P. F. (1970) The electroencephalogram in herpes simplex encephalitis. Lancet, 2, 525.

UPTON. A. \& GUMPERT, J. (1970) Electroencephalography in diagnosis of herpes simplex encephalitis. Lancet, i, 650. 